\title{
Pathologic and Electrocardiographic Findings in Sudden Cardiac Death in Racehorses
}

\author{
Keiji KIRYU, Noboru MACHIDA*, Yoko KASHIDA, Toyohiko YOSHIHARA ${ }^{1)}$, Akio AMADA ${ }^{2)}$ and \\ Takeshi YAMAMOTO ${ }^{3)}$ \\ Department of Veterinary Pathology, Tokyo University of Agriculture and Technology, Fuchu, Tokyo 183-8509, ${ }^{1)}$ Equine Research \\ Institute, Japan Racing Association (JRA), Utsunomiya, Tochigi 320-0856, ${ }^{2}$ Blood Horse Training Center, JRA Foundation, Minato-ku, \\ Tokyo 105-0001, and ${ }^{3)}$ Racing Museum, JRA, Fuchu, Tokyo 183-0024, Japan
}

(Received 4 March 1999/Accepted 25 April 1999)

ABSTRACT. Five racehorses in apparently normal condition succumbed to sudden cardiac death (SCD) during or shortly after intensive training exercise. Cardiopathologic examination was performed. In 1 of the 5 horses, the use of an electrocardiogram (ECG) recording taken continuously for $440 \mathrm{sec}$ enabled us to analyze some of the arrhythmias in the terminal event of SCD. The ECG tracing exhibited the R-on-T phenomenon following a pair of ventricular premature contractions (VPCs). The phenomenon rapidly degenerated into ventricular fibrillation, which led to cardiac arrest. In all 5 horses cardiopathologic examination revealed the following lesions: (i) foci of myocardial fibrosis in the right atrium located close to the sinoatrial (SA) node, (ii) fibrotic and/or fibroplastic changes in the upper portion of the interventricular septum, including the atrioventricular (AV) conduction system, and (iii) arterio- and arteriolosclerosis of the SA and AV node vessels. Pathogenetically, the process by which the focal lesions of myocardial ischemia secondary to vascular sclerosis progressed into fibrosis and/or fibroplasia could play a major role in the genesis of arrhythmias. Presumably the fibrotic and/or fibroplastic changes in the area of the AV bundle and its bundle branches are closely related to the onset of fatal ventricular arrhythmias such as VPCs, deteriorating into ventricular fibrillation. SCD in training and racing Thoroughbred horses appears to be due to arrhythmia.-KEY wORDS: arrhythmia, equine, heart, pathology, sudden death.

J. Vet. Med. Sci. 61(8): 921-928, 1999

Sudden death (SD) in the horse is frequently attributed to cardiac disease [4, 12, 30], although pathologic surveys of equine SD have shown no identifiable cause of arrhythmic death [10, 29, 31], which is regarded as inexplicable [24]. In human medicine, sudden cardiac death $(\mathrm{SCD})$ has been described in detail by many investigators. In humans, SCD generally denotes death that is non-violent or non-traumatic, unexpected, witnessed and occurs within a few minutes ('instantaneous death') or up to a maximum of $1 \mathrm{hr}$ after the onset of an abrupt change in clinical status [33]. Most human SCD cases are assumed to be due to acute fatal arrhythmias - ventricular tachycardia or ventricular fibrillation [3, 11, 23, 33, 36].

Spontaneous arrhythmias are common in the horse [29], but there have been no systematic pathologic investigations dealing with arrhythmias, except for one study of horses and mules over 10 years of age [27]. We have made some observations on cardiac pathology in Thoroughbred horses under 10 years old that were affected with various arrhythmias, including atrial fibrillation (AF) [1, 16-19]. These cardiopathologic studies of arrhythmias have been used as the basis of a proposed correlation between the pathogenesis of arrhythmia and SCD in the racehorse [20, 22]. However, as in the case of arrhythmic death in the athlete [26], we have had difficulty proving conclusively that equine SD is definitely caused by an arrhythmia. No

\footnotetext{
* Correspondence to: Machida, N., Department of Veterinary Pathology, Tokyo University of Agriculture and Technology, Fuchu, Tokyo 183-8509, Japan.
}

matter what conclusions have been drawn thus far about human SCD, the precise relationship between the prodromal signs (including fatal arrhythmias) and the pathologic condition is still controversial $[3,5,8,9,11,23,26,36]$.

This paper reports the cardiopathologic changes found in 5 racehorses that had been in apparently normal condition when they succumbed to SCD or instantaneous death during or shortly after intensive training exercise. It also describes the terminal electrocardiographic events in 1 of the 5 animals.

\section{MATERIALS AND METHODS}

Necropsy of the 5 racehorses in this study (Table 1) was performed as soon as possible after SD, usually within $3 \mathrm{hr}$. Fortuitously, when horse 1 fell and lay down suddenly shortly after a training exercise, it was possible to record an electrocardiogram (ECG). For the ECG recording, a baseapex lead was used [29]; the positive electrode was positioned over the apex-beat area of the heart and the negative electrode over the cardiac base. The recording was performed continuously over the 400 -sec terminal event of the SD, at a paper speed of 25 or $50 \mathrm{~mm} / \mathrm{sec}$. So that we could analyze the arrhythmias, the onset of the R-on-T phenomenon was set up as a 0 -sec point, and the beginning point of the recording was calculated back to $370 \mathrm{sec}$ (Table 2). For measurements of the Q-T interval, 5 successive intervals with sinus rhythm were randomly taken from the ECG tracing, mainly at a paper speed of $50 \mathrm{~mm} / \mathrm{sec}$. The corrected Q-T interval (QTc) was calculated using Coryicelli's formula (QTc $=0.36 \sqrt{\mathrm{RR}} \pm 0.07 \mathrm{sec})$. 
Table 1. Five Thoroughbred racehorses that suffered sudden cardiac death during or shortly after intensive training exercise

\begin{tabular}{ccccl}
\hline Horse no. & Sex $^{\text {a) }}$ & Age $(\mathrm{yr}, \mathrm{mo})$ & Racing history & SCD $^{\text {b) }}$ during/after training \\
\hline 1 & F & 2,5 & No & Shortly after \\
2 & M & 3,0 & Yes & During \\
3 & F & 3,6 & Yes & Shortly after \\
4 & M & 6,0 & Yes & During \\
5 & M & 7,8 & Yes $^{\text {d) }}$ & During \\
\hline
\end{tabular}

a) F, female; M, male. b) SCD, sudden cardiac death. c) Death occurred about 8 min after the onset of an abrupt change in clinical status in horse 1, and after a few minutes in the others. d) Sudden loss of speed during a race, twice (1st: unknown cause; 2 nd: epistaxis).

Table 2. Electrocardiographic findings in the terminal event of sudden death in a racehorse (horse 1)

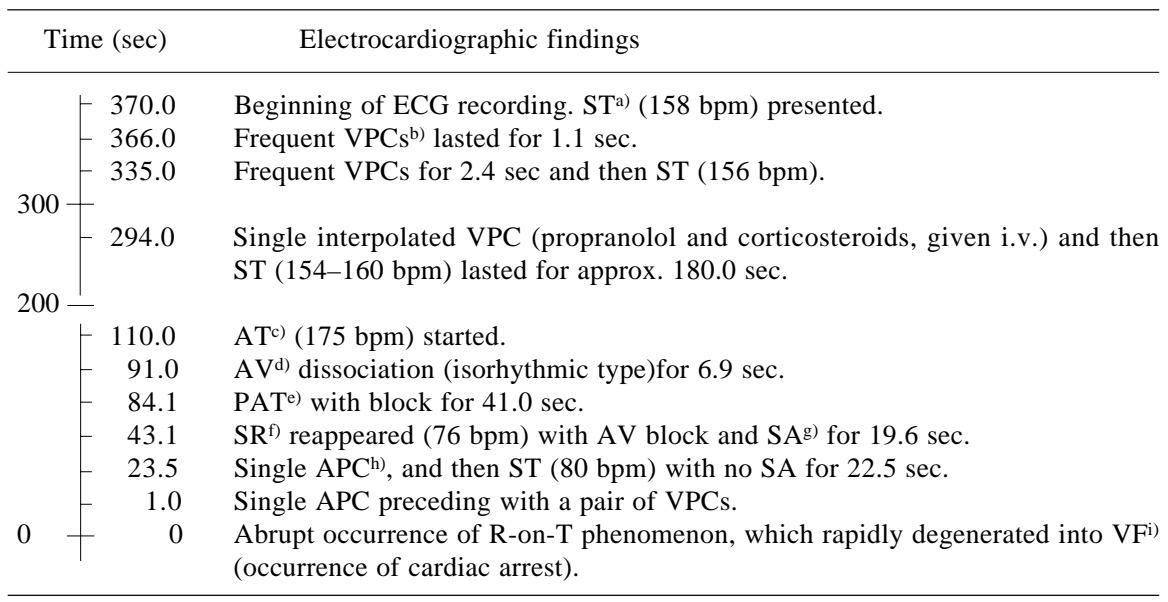

a) ST, sinus tachycardia. b) VPC, ventricular premature contraction. c) AT, atrial tachycardia. d) AV, atrioventricular. e) PAT, paroxysmal atrial tachycardia. f) SR, sinus rhythm. g) SA, sinus arrhythmia. h) APC, atrial premature contraction. i) VF, ventricular fibrillation.

Histopathologic examination of the 5 horses focused on the hearts in the same manner described in our previous reports [16-22]. Special care was taken to retain the correct sequence of tissue block during histologic processing so that both the topography of the conduction system and the arterial supply of the sinoatrial and atrioventricular (SA and AV) nodes could be reconstructed from the previous reports $[17,20,21]$. All sections were stained with both hematoxylin and eosin and elastica van Gieson. Where necessary, serial sections were stained with Masson's trichrome, alcian blue and Wilder's method for reticulum.

\section{RESULTS}

Clinical features: All the 5 racehorses fell and lay down suddenly during or shortly after a very strenuous training exercise. In horses 2 to 5, SD occurred within a few minutes after the unexpected onset of abnormal signs, but SD took approximately $8 \mathrm{~min}$ in horse 1 . Horses 1 to 4 had no history of cardiac problems and had been asymptomatic during prior vigorous training and/or racing. Horse 1 had no racing history. Horse 5 had twice shown a sudden loss of speed during a race (Table 1).

All of the horses had certain clinical features in common, and their deaths were all witnessed. There was extreme respiratory distress, abnormal and rapid breathing with occasional deep expirations, profuse sweating all over the body and agonal trembling. Apart from horse 1, all were dead before emergency veterinary treatment could be given, although a veterinarian was on emergency duty. In the case of horse 1, the ECG recording was used successfully to analyze the arrhythmias. Ventricular premature contractions (VPCs) were observed (see below), and 2 drugs were administered intravenously (Table 2). One was the adrenoreceptor antagonist ( $\beta$-blocker), propranolol hydrochloride, and the other a corticosteroid, betamethasone disodium phosphate. The horse weighed $413 \mathrm{~kg}$; a total dose of $20 \mathrm{mg}$ of each drug was given.

ECG findings: As summarized in Table 2, sinus tachycardia (ST) presented first, and finally terminated in loss of cardiac function through ventricular fibrillation (VF). Until the onset of VF, however, the 370-sec tracing exhibited 
a variety of interrupted arrhythmias. They were, in turn, VPCs (Fig. 1-a), atrial tachycardia (AT), AV dissociation, paroxysmal atrial tachycardia (PAT) with block (Figs. 1-b and 1-c), returning abruptly from PAT with block to sinus rhythm (Fig. 1-d), an atrial premature contraction (APC), and VPCs followed by the R-on-T phenomenon that degenerated into VF, leading to sudden cardiac arrest (Fig. $1-e)$.

At the last 1-sec point, an APC occurred abruptly (Fig. 1e), although the tracing until this point had continued with a sinus rhythm (P-P and R-R intervals, both $0.74 \mathrm{sec}$; QTc: $0.32 \mathrm{sec})$. The $\mathrm{P}$ wave was abnormally peaked, and the coupling interval was $0.46 \mathrm{sec}$; it was distinctly short, compared with that $(0.56 \mathrm{sec})$ of the previous APC (Table 2 ). The APC at the 1-sec point was accompanied by a relatively wide $\mathrm{T}$ wave and a long QTc $(0.44 \mathrm{sec})$. Except for this prolongation, there was no evidence that the QTc increased significantly in the whole ECG tracing. This APC was followed by a sequence of 2 VPCs (R-R interval: 0.32 $\mathrm{sec})$. This pair of VPCs exhibited an R-on-T phenomenon at the 0 -sec point, and rapidly deteriorated into VF. The second VPC appeared to encroach on the preceding $\mathrm{T}$ wave ending: not before the apex of the $\mathrm{T}$ wave, but after it. The ECG recording was continued until the paper ran out 440 sec after recording had started. The above observations confirmed that a fatal arrhythmia had led to the cardiac arrest and SD.

Gross findings: At necropsy, all 5 racehorses had no recognizable gross heart lesions, but particular emphasis on the acute systemic circulatory disturbances common to the animals revealed visceral congestion and subpleural hemorrhages. The hemorrhages exhibited sporadic petechiae and/or occasional ecchymoses, which were scattered in the parietal and visceral subpleurae and varied in severity and nature with each case.

Microscopic cardiac findings: All 5 horses had certain cardiohistopathologic changes in common (Table 3), although there were no hemorrhagic findings in the lungs except for the subpleural tissues. The cardiac changes were characterized by foci of myocardial fibrosis located in the

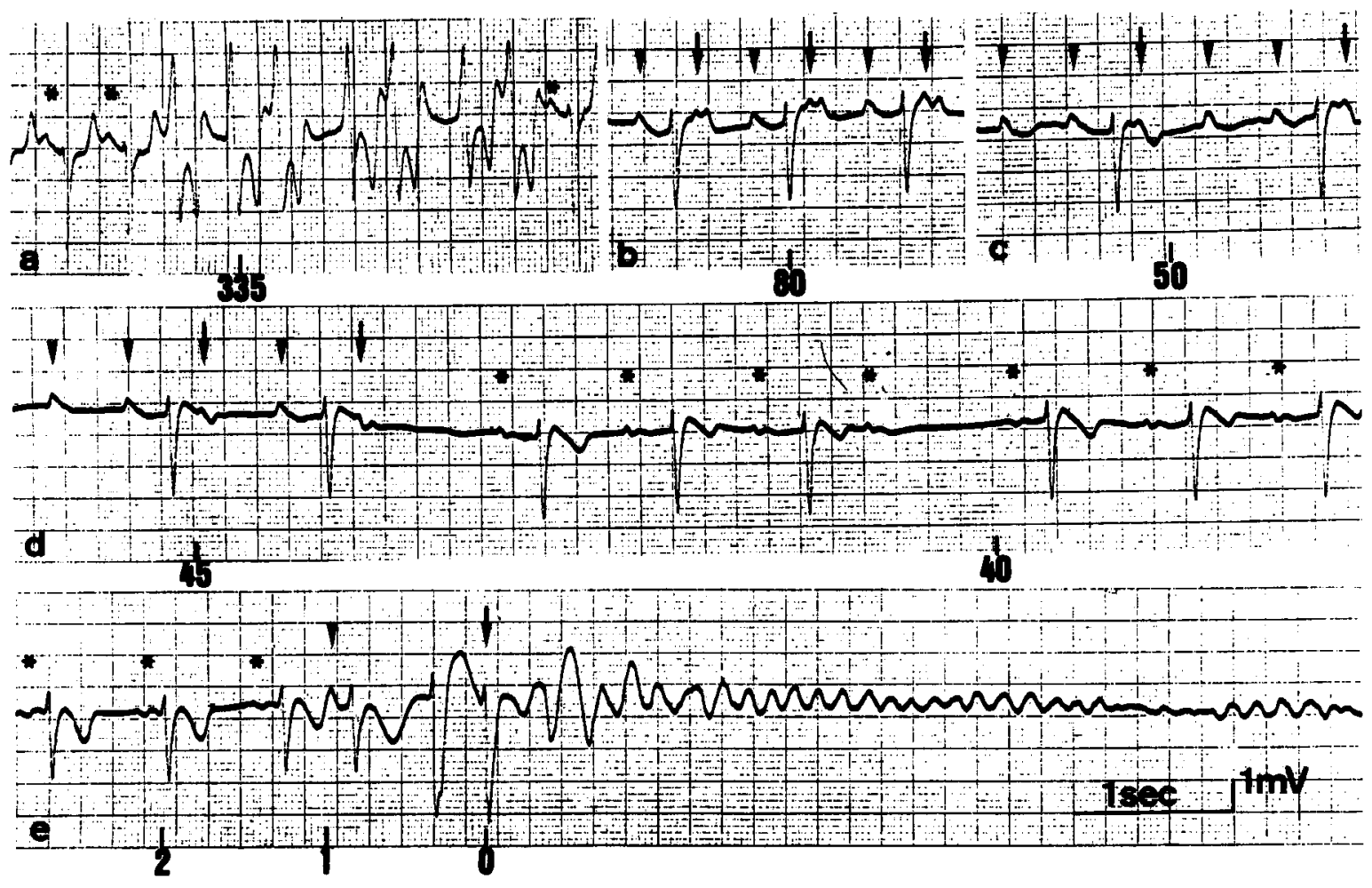

Fig. 1. Electrocardiographic tracing in the terminal event of sudden cardiac death in horse 1. The numbers at the bottom of each tracing (Figs. 1-a to 1-e) indicate the time point before the R-on-T phenomenon, which was counted as 0 sec. (a) Frequent ventricular premature contractions (VPCs) consisting of a single VPC and a pair of VPCs. Asterisks: sinus P wave. (b) Paroxysmal atrial tachycardia (PAT) with 2:1 atrioventricular (AV) block. The conducted P wave (arrowheads) and the nonconducted wave (arrows) superimposed on the T wave appear one after the other. (c) PAT with 3:1 block, showing the peaked P wave (arrowheads) and the P wave (arrows) superimposed on the T wave. (d) Returning abruptly from PAT with AV block to sinus rhythm, leaving the Wenckebach phenomenon. Note the change from the peaked $\mathrm{P}$ wave (arrowheads) and the wave (arrows) that is superimposed on the T wave in PAT to the diphasic (+/-) or vibratory (+///+) P wave (asterisks) in sinus rhythm. (e) Terminal electrocardiographic event. Sinus rhythm (asterisks: sinus $\mathrm{P}$ wave) changes abruptly into atrial premature contraction (arrowhead), a pair of VPCs, the R-on-T phenomenon (arrow) and ventricular fibrillation. 
Table 3. Histopathologic changes in the right atrium, interatrial septum, atrioventricular junction and upper portion of the interventricular septum of Thoroughbred racehorses suffering sudden cardiac death

\begin{tabular}{|c|c|c|c|c|c|c|}
\hline \multirow{2}{*}{ Horse no. ${ }^{\text {a) }}$} & \multicolumn{4}{|c|}{ MF in right atrium and Ias ${ }^{\mathrm{b})}$} & \multirow{2}{*}{$\frac{\mathrm{AS}^{\mathrm{b})}}{\mathrm{SANa} / \mathrm{AVNa}}$} & \multirow{2}{*}{$\frac{\mathrm{FP} \text { and/or } \mathrm{MF}^{\mathrm{b})}}{\mathrm{AVB} / \mathrm{LBB} / \mathrm{RBB}}$} \\
\hline & Ps1 & An1 & Med & Ias & & \\
\hline 1 & _c) & $+^{c)}$ & + & + & $+/++^{c)}$ & $++/++/-$ \\
\hline 2 & ++ & ++ & + & ++ & $++/+$ & $+1-1++$ \\
\hline 3 & - & + & + & - & $-1+$ & $+1-1-$ \\
\hline 4 & - & + & + & - & $-/++$ & $++/+/+$ \\
\hline 5 & + & + & + & - & $+/+$ & $+/++/-$ \\
\hline
\end{tabular}

a) Body weight of 5 horses, 400-450 kg; Ratio of heart wt. to body wt., 0.00800.0093. b) MF, focal myocardial fibrosis; Ias, interatrial septum; AS, arteriosclerotic changes; FP, fibroplasia; Psl, posterolateral region; Anl, anterolateral region; Med, medial region; SANa, sinoatrial node artery; AVNa, atrioventricular node artery; AVB, AV bundle; LBB, left bundle branch; RBB, right bundle branch. c) ++, severe lesion; +, mild lesion; -, no lesion.

right atrium, the interatrial septum and the upper portion of the interventricular septum, including the AV conduction system (Figs. 2-5): the AV node, the AV bundle and the left bundle branch (LBB) and right bundle branch (RBB). Also, a fibroplastic response was observed in the summit area of the interventricular septum, which is rich in connective tissue. These fibrotic and/or fibroplastic lesions were most often seen around or adjacent to the blood vessels, which frequently showed degenerative and sclerotic changes. The vascular changes described below (Figs. 6 and 7) were always present in the AV node artery and occasionally in the SA node artery.

The foci of myocardial fibrosis were classified into two grades: mild and severe. Mild lesions were minute compared with severe lesions. Mild lesions were characterized by a loose or relatively dense proliferation of collagen fibers surrounding atrophic muscle and indicating interstitial fibrosis. Severe lesions were large and characterized by an extensive loss of muscle fibers, which had been replaced by dense collagen fibers, indicating progressive and substitutive fibrosis (or replacement fibrosis). These lesions were occasionally accompanied by adipose tissue. The mild and/or severe fibrotic lesions were found close to the SA node, located in the anterolateral and medial regions of the right atrium (Fig. 2), frequently in the LBB area (Fig. 4), and occasionally in the RBB (Fig. 3) and interatrial septum. In the myocardium bordering the fibrotic lesions, the peri- and endomysial reticulum fibers appeared to fuse and blend with the collagen fibers, fading away in the fibrous strands within the lesions (Fig. 5).

Morphologically, the AV node, the AV bundle and the bundle branches (BBs) extended anteriorly and downwards in the upper portion of the interventricular septum. This conduction system was located below the cardiac cartilage (Fig. 6). The specialized muscle fibers were embedded in proliferative collagen fibers in the AV bundle (Fig. 3). The dense fibrous tissue in the interstitium showed fibroplasia arising from the fibrous ring including fibrous trigone, which was also fibroplastic in the summit of the interventricular septum. Occasionally the BBs were also affected by fibroplasia. The fibroplastic or fibrous strands extended from the AV bundle and its BBs into the myocardial interstitium, and the strands were often part of the fibrotic foci of the mild or severe lesions (Figs. 3-5).

Within and around both the fibrotic and fibroplastic lesions mentioned above, degenerative and nonatherosclerotic vascular changes were found in the walls of the small muscular arteries and arterioles (Figs. 6 and 7). These vessels exhibited a variety of sclerotic changes. The sclerotic changes included thickening of both the intima of the small arteries and the full walls of the swollen arterioles. The former exhibited a proliferation of collagen fibers and/ or muscle fibers (myocellular proliferation) in the intima: the tremendously thickened intima was associated with fragmentation or anomalous irregular thickening of the internal elastic lamina. The latter showed edematous changes, with intercellular accumulation of a slightly eosinophilic and alcian blue-positive fluid, as well as collagen proliferation. Some vessels were frequently accompanied by increased numbers of elastic fibers in the intima and/or media. Rarely, small arteries had aneurysms in their walls, showing proliferative intimal thickening, and medial and adventitial fibrosis (Fig. 6). Occasionally, those vascular walls that had thickened media demonstrated muscular hyperplasia, which assumed a longitudinal disposition in the outer media, accompanied by perimedial proliferation of collagen fibers.

\section{DISCUSSION}

In all 5 horses, the gross necropsy findings of visceral congestion and subpleural petechiae and/or ecchymoses were associated with the agonal hemorrhages that arose secondary to acute cardiac failure and severe hypoxemia. These findings, plus the ECG detection of a variety of arrhythmias in the terminal event of horse 1, are explainable in terms of cardiac arrest rather than death from pulmonary hemorrhage $[4,14]$. Since the only hemorrhagic findings in 


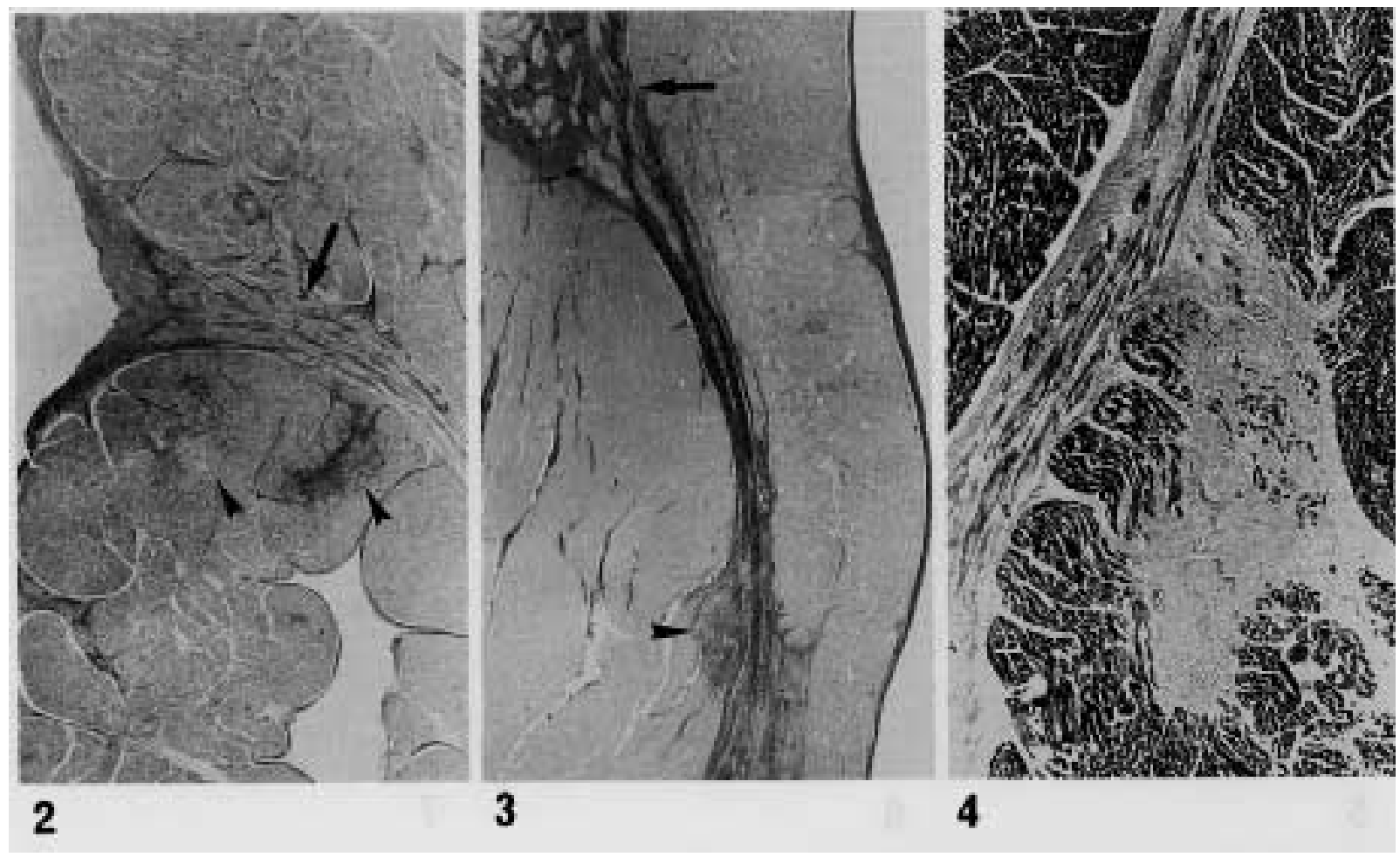

Fig. 2. Foci of myocardial fibrosis (arrowheads) in the anterolateral region of the right atrium, close to the sinoatrial node (arrow). Horse 2. Elastica van Gieson $(E V G)$ stain, $\times 10$.

Fig. 3. The AV bundle affected by fibroplasia (arrow) and a focal lesion of myocardial fibrosis (arrowhead) close to the right bundle branch. Horse 2. EVG stain, $\times 10$.

Fig. 4. Focal lesion of myocardial fibrosis close to the left bundle branch. Horse 1 . Masson's trichrome stain, $\times 32$.

the lungs were in the subpleural tissues, we can assume that the hemorrhages were due to strenuous but fruitless agonal attempts to breathe during cardiac failure or dysrhythmia. In addition, concerning the emergency treatment for the cardiac failure in horse 1, it was preferable to have used corticosteroids and propranolol as soon as frequent AVCs were detected. Probably, the administration of such drugs must have delayed advancing to the terminal event.

Electrocardiographically, the abrupt and fatal arrhythmias, a single APC and a pair of VPCs in series before the onset of VF in horse 1 somewhat resembled the ECG findings of human SCD [5, 23, 37]. The QRS complex of the VPCs appeared to fall in the preceding period of relatively increased ventricular vulnerability. This alteration in the regional refractory period may have been associated with the preceding APC, which occurred abruptly and was accompanied by a wide T wave and long QTc. Accordingly, the R-on-T phenomenon [31] occurred and rapidly degenerated into VF, which led to arrhythmic death $[3,11$, 23, 33, 36]. Therefore, the SCD in this training Thoroughbred horse can be categorized clearly by the complete ECG recording as an arrhythmic death, although we have confirmed one racehorse with SCD that exhibited ventricular tachycardia (VT) in part of the terminal episode [20]. The ECG findings of the other horses (horses 2 and 5) could have been compatible with those of the VPCs-affected horse 1 or the VT-affected horse [20].

In the racehorse, AF, APCs or VPCs occasionally occur during or shortly after the stress of a race [29]. In particular, the occurrence of paroxysmal AF causes the horse to slow down during the race $[2,22]$. It is known that human $\mathrm{AF}$ can precipitate heart failure, angina and ventricular arrhythmia, and increases cardiovascular mortality and the rate of SCD [7, 34]. On the other hand, no relation has been demonstrated between AF and SCD in the horse [10, 29]. We have found that horses that have recovered from paroxysmal AF frequently reveal exercise-induced VPCs on the radiotelemetric ECG [22]. By contrast, apparently healthy horses without a history of AF have no arrhythmias during exercise [35].

The pathologically significant findings common to all 5 racehorses in the present study were as follows: (i) the atrial lesions of myocardial fibrosis located close to the SA node, (ii) sclerotic vascular changes of the AV and SA node arteries, and (iii) fibroplastic and/or fibrotic lesions in the upper portion of the interventricular septum, including the $\mathrm{AV}$ bundle and BBs. There were no signs of the congenital abnormalities or cardiomyopathy sometimes found in humans $[3,26]$. The findings of this study closely resembled those observed in racehorses with $\mathrm{AF}[1,18,22]$ and/or the exercise-induced VPCs [22] and SCD [20] we reported previously. 


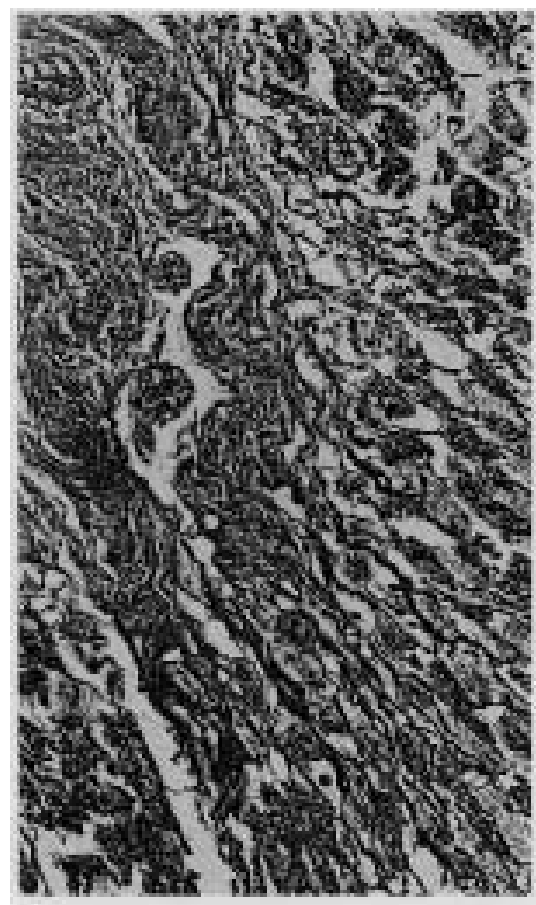

5

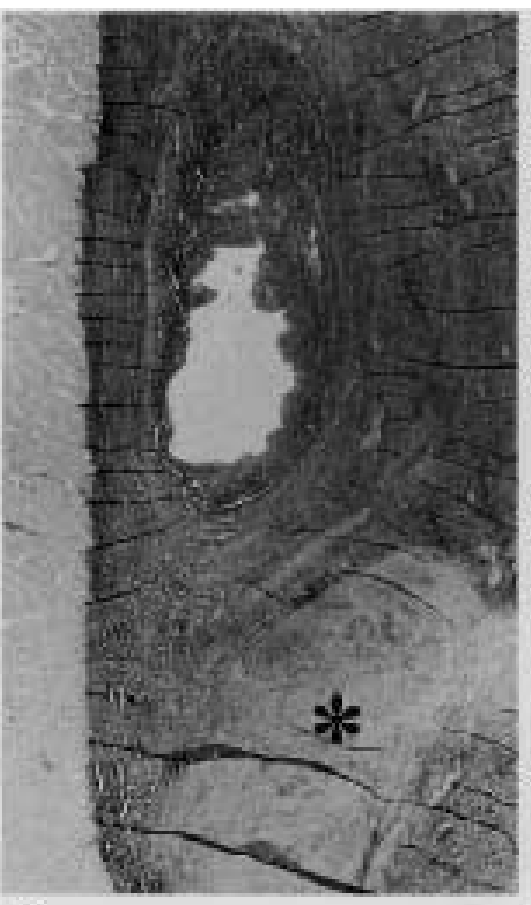

6

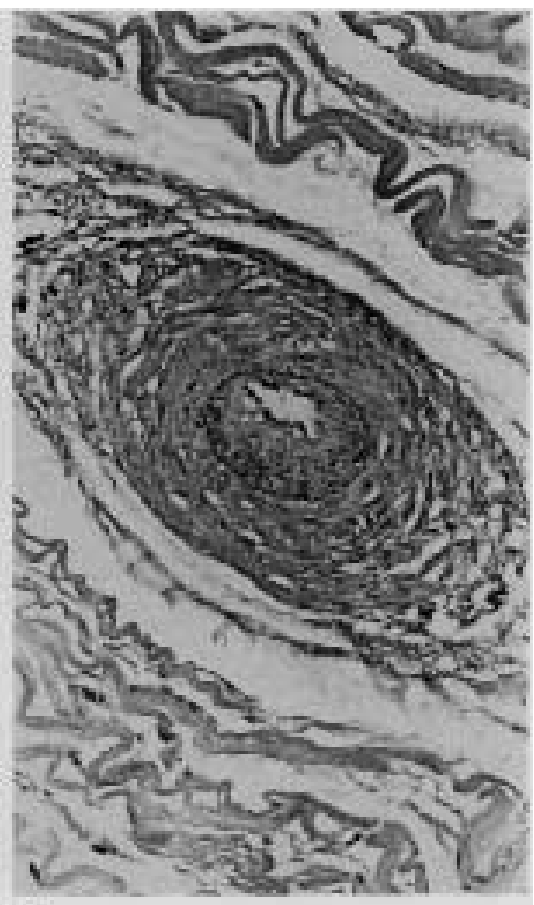

7

Fig. 5. Reticulum fibers around the focal lesion in Fig. 4 appearing to fuse and blend with collagen fibers. Wilder's reticulum stain, $\times$ 135.

Fig. 6. Small artery located in intimate contact with the cardiac cartilage (asterisk). Note the aneurysm wall showing proliferative intimal thickening and medial and adventitial fibrosis. Horse 1 . EVG stain, $\times 13$.

Fig. 7. Small artery within the AV node (AV node artery), showing the thickened intima and narrowed lumen. Note the nodal fibers in the vicinity of the artery. Horse 5. EVG stain, $\times 268$.

Likewise, the pathogenesis of the above-mentioned lesions was presumed to be basically the same as that of the cardiopathologic changes found in other horses with various arrhythmias and SCD [1, 16-18, 20, 22]. One of the findings that had emerged previously was the sclerotic change in the SA node artery. This indicates intramyocardial microvascular changes, including arterioand arteriolosclerosis, which impair the ischemic contiguity of the SA junction, inducing myocardial fibrosis in the atrium and suggesting the possibility of ensuing AF. As this study in particular indicated, the sclerotic SA node artery could bring about local circulatory disturbances not only in the right atrium and interatrial septum, but also in the AV node area [22]. This is likely because in the horse the atrial branches of the coronary artery, comprising the SA node artery [17], which receives a dual blood supply $[20,21]$ from (i) the left circumflex branch and (ii) the trunk of the right coronary artery, give off further branches into the AV node area, including the AV bundle and BBs. Furthermore, the equine AV node artery receives a triple blood supply [21] from (i) the above-mentioned branches of the SA node artery, (ii) the anterior septal branch of the paraconal interventricular branch, and (iii) the posterior septal branches of both the right circumflex branch and subsinual interventricular branch. So, theoretically, regardless of the condition of the SA node artery, the ischemic contiguity of the AV node area could be compromised by sclerotic changes in branches (ii) and/or (iii). In brief, chronic or insidious and recurrent ischemia may induce fibrotic and/or fibroplastic lesions in the upper portion of the interventricular septum, including the AV conduction system. We emphasize that this progression from ischemia to fibrosis and/or fibroplasia could play an important role in creating a suitable scenario for an arrhythmogenic condition [22], which could have given rise to VPCs in the present horses. It is interesting that such sclerotic changes in the arteries supplying the SA and AV nodes also occur in humans, and their role in SCD is likely to come under scrutiny $[6,15]$.

According to the study of microscopic lesions in the hearts of 23 non-Thoroughbred horses over 10 years old [27], arteriosclerosis and myocardial fibrosis were sometimes found in the interventricular septum, with and without arrhythmias. In our previous observations of 19 young Thoroughbred racehorses between 1 year and 3 months and 5 years and 11 months old that exhibited no arrhythmias [19], the area of the AV conduction system indicated no fibrosis, fibroplasia or arteriosclerosis. Furthermore, in support of our previous case report [25], the myocardial fibrosis located close to the $\mathrm{BBs}$ in the 
present study coincided with that detected in a 20 -year-old pony mare with persistent VT that led to cardiac arrest. In the case of racehorses, it is reasonable to suppose that there is a relationship between the genesis of instantaneous death and the fibrotic and/or fibroplastic lesions in the areas of the AV bundle and BBs, regardless of age $[25,27]$, in the same way that there is a relationship between instantaneous death and congenital abnormalities in the human heart $[3$, 26]. Humans with LBB and/or RBB block who have no clinical evidence of cardiovascular disease have an increased risk of developing overt cardiovascular disease and SCD [5, $9,11,23]$. The equivalent of the cardiopathologic changes related to $\operatorname{LBB}[11,23]$ and $\operatorname{RBB}[5,9,28]$ block in humans, however, would be clinically occult in a routine equine ECG $[13,29]$.

As a consequence of the above findings, the 5 racehorses were diagnosed pathologically as having SCD, and a diagnosis of arrhythmic death was made electrocardiographically in 1 of the animals. The results presented here recognize the pathology that places horses at risk for arrhythmias, and suggest the possibility of using the racehorse for comparative cardiology, especially in the study of arrhythmic death in young competitive athletes.

ACKNOWLEDGMENTS. We thank Drs. Y. Fujita, Y. Imuro and T. Seki for their cooperation while they were veterinary students at Tokyo University of Agriculture and Technology. This study was supported in part by a grantin-aid from the Equine Research Institute, Japan Racing Association.

\section{REFERENCES}

1. Amada, A. and Kiryu, K. 1987. Atrial fibrillation in the race horse. Heart Vessels (Suppl.) 2: 2-6.

2. Amada, A. and Kurita, K. 1975. Five cases of paroxysmal atrial fibrillation in the racehorse. Exp. Rep. Equine Health Lab. 12: 89-100.

3. Bharati, S. and Lev, M. 1994. Sudden death in athletes conduction system: Practical approach to dissection and pertinent pathology. Cardiovasc. Pathol. 3: 117-127.

4. Brown, C. M., Kaneene, J. B. and Taylor, R. F. 1988. Sudden and unexpected death in horses and ponies of 200 cases. Equine Vet. J. 20: 99-103.

5. Brugada, P. and Brugada, J. 1992. Right bundle branch block, persistent ST segment elevation and sudden cardiac death: A distinct clinical and electrocardiographic syndrome. A multicenter report. J. Am. Coll. Cardiol. 20: 1391-1396.

6. Burke, A. P., Subramanian, R., Smialek, J. and Virmani, R. 1993. Nonatherosclerotic narrowing of the atrioventricular node artery and sudden death. J. Am. Coll. Cardiol. 21: 117122.

7. Camm, A. J. and Obel, O. A. 1996. Epidemiology and mechanism of atrial fibrillation and atrial flutter. Am. J. Cardiol. 78(8A): 3-11.

8. Consensus Statement of The Joint Steering Committees of The Unexplained Cardiac Arrest Registry of Europe and of Idiopathic Ventricular Fibrillation Registry of The United States. 1997. Survivors out-of-hospital cardiac arrest with apparently normal heart. Circulation 95: 265-272.
9. Corrado, D., Nava, A., Buja, G., Martini, B., Fasoli, G., Oselladore, L., Turrini, P. and Thiene, G. 1996. Familial cardiomyopathy underlies syndrome of right bundle branch block. ST segment elevation and sudden death. J. Am. Coll. Cardiol. 27: 443-448.

10. Deem, D. A. and Fregin, G. F. 1982. Atrial fibrillation in horses: A review of 106 clinical cases, with consideration of prevalence, clinical signs, and prognosis. J. Am. Vet. Med. Assoc. 180: 261-265.

11. Fahy, G. J., Pinski, S. L., Miller, D. P., McCabe, N., Pye, C., Walsh, M. J. and Robinson, K. 1996. Natural history of isolated bundle branch block. Am. J. Cardiol. 77: 1185-1190.

12. Gelberg, H. B., Zachary, J. F., Everitt, J. I., Jensen, R. C. and Smetzer, D. L. 1985. Sudden death in training and racing Thoroughbred horses. J. Am. Vet. Med. Assoc. 187: 13541356.

13. Glazier, D. B., Littledike, E. T. and Cook, H. M. 1983. The electrocardiographic changes in experimentally induced bundle branch block in the equine heart. Irish Vet. J. 37: 71-76.

14. Gunson, D. E., Sweeney, C. R. and Soma, L. R. 1988. Sudden death attributable to exercise-induced pulmonary hemorrhage in racehorses: Nine cases (1981-1983). J. Am. Vet. Med. Assoc. 193: 102-106.

15. James, T. N. 1990. Morphologic characteristics and functional significance of focal fibromuscular dysplasia of small coronary arteries. Am. J. Cardiol. 65: 12G-22G.

16. Kiryu, K. 1980. Cardiopathology of arrhythmias in the horse. Proc. Am. Assoc. Equine Practnrs. 26: 457-468.

17. Kiryu, K., Kaneko, M., Kanemaru, T., Yoshihara, T., Hasegawa, M. and Tomioka, Y. 1985. Cardiopathology of sinoatrial block in horses. Jpn. J. Vet. Sci. 47: 45-54.

18. Kiryu, K., Kaneko, M., Oikawa, M., Kanemaru, T., Yoshihara, T. and Satoh, H. 1977. Histopathogenesis of atrial fibrillation in the horse: Cardiopathology of an additional case. Exp. Rep. Equine Health Lab. 14: 54-63.

19. Kiryu, K., Kaneko, M., Yoshihara, T., Hasegawa, M. and Tomioka, Y. 1981. Microscopic observation on the heart in horses which exhibited no arrhythmias. Bull. Equine Res. Inst. 18: 141-147.

20. Kiryu, K., Nakamura, T., Kaneko, M., Oikawa, M. and Yoshihara, T. 1987. Cardiopathology of sudden cardiac death in the race horse. Heart Vessels (Suppl.) 2: 40-46.

21. Kiryu, K., Nakamura, T., Machida, N. and Mukai, S. 1994. Blood supply of the sinoatrial and atrioventricular node arteries in the Thoroughbred racehorse. Adv. Anim. Cardiol. 27: 60-69 (in Japanese with English summary).

22. Kiryu, K., Nakamura, T., Machida, N., Tangiku, M., Kaneko, M., Yoshihara, T. and Yamamoto, T. 1991. Cardiopathological changes in racehorses with abrupt deceleration of speed during a race. Jpn. J. Equine Sci. 2: 21-30.

23. Leenhardt, A., Glaser, E., Burguera, M., Nurnberg, M., Maison-Blancke, P. and Cumel, P. 1994. Short-coupled variant of torsade de pointes. A new electrocardiographic entity in the spectrum of idiopathic ventricular tachyarrhythmias. Circulation 89: 206-215.

24. Luck, V. M. 1987. Sudden death. Equine Vet. J. 19: 85-86.

25. Machida, N., Nakamura, T., Kiryu, K., Haramaki, S. and Too, K. 1992. Cardiopathological observation in a case of persistent ventricular tachycardia on a pony mare. J. Vet. Med. Sci. 54: 1213-1216.

26. Maron, B. J., Shirani, J., Poliac, L. C., Mathenge, R., Roberts, W. C. and Mueller, F. O. 1996. Sudden death in young competitive athletes. Clinical, demographic, and pathological 
profiles. J. Am. Med. Assoc. 276:199-204.

27. Marcus, L. C. and Ross, J. N. Jr. 1967. Microscopic lesions in the hearts of aged horses and mules. Pathol. Vet. 4: 162 185.

28. Martini, B., Nava, A., Thiene, G., Buja, G. F., Canciani, B., Scognamiglio, R., Daliento, L. and Volta, S. D. 1989. Ventricular fibrillation without apparent heart disease: Description of six cases. Am. Heart J. 118: 1203-1209.

29. Patteson, M. W. 1996. Equine Cardiology, Blackwell Sci. Ltd., Oxford.

30. Platt, H. 1982. Sudden and unexpected death in horses: A review of 69 cases. Br. Vet. J. 138: 417-429.

31. Reimer, J. M., Reef, V. B. and Sweeney, R. W. 1992. Ventricular arrhythmia in horses: 21 cases(1984-1989). J. Am. Vet. Med. Assoc. 201: 1237-1243.

32. Report of a World Health Organization Scientific Group. 1985. Sudden Cardiac Death. World Health Organization, Geneva.

33. Roberts, W. C. 1986. Sudden cardiac death: Definitions and causes. Am. J. Cardiol. 57: 1410-1413.

34. Rostagno, C., Bacci, F., Martelli, M., Naldoni, A., Bertini, G. and Gensini, G. 1995. Clinical course of lone atrial fibrillation since first symptomatic arrhythmic episode. Am. $J$. Cardiol. 76: 837-839.

35. Scheffer, C. W. J., Robben, J. H. and Sloet van Oldruitenborgh-Oosterbaan, M. M. 1995. Continuous monitoring of ECG in horses at rest and during exercise. Vet. Rec. 137: $371-374$.

36. Shen, W.-K., Edwards, W. D., Hammill, S. C., Bailey, K. R., Ballard, D. J. and Gersh, B. J. 1995. Sudden unexpected nontraumatic death in 54 young adults: A 30-year population-based study. Am. J. Cardiol. 76: 148-152.

37. Wang, F.-S., Lien, W.-P., Fong, T.-E., Lin, J.-L., Cherng, J. J., Chen, J.-H. and Chen, J.-J. 1986. Terminal cardiac electrical activity in adults who die without apparent cardiac disease. Am. J. Cardiol. 58: 491-495. 